\title{
Criterios y métodos para seleccionar la ubicación de los rellenos sanitarios
}

\section{Criteria and methods for selecting the location of the landfill}

\author{
Carlos Alberto Canelo Dávila ${ }^{1,2^{*}}$ (i)
}

\section{RESUMEN}

La selección del lugar en que se construirá un relleno sanitario es un proceso complejo, que requiere la asistencia de expertos en la disposición final de residuos y analistas de datos, la inclusion de los actores sociales y politicos así como el uso de métodos de apoyo a la toma de decisiones. El objetivo del presente artículo es responder a la pregunta: ¿Qué criterios y métodos se pueden emplear para la selección de un sitio de ubicación en el que se construya un relleno sanitario? Como respuesta se ha sistematizado los criterios de análisis según los factores socio-económicos, ambientales, físicos y técnicos. También se ha realizado una clasificación de los métodos MCDM más importantes que pueden ser empleados por los analistas para el apoyo a las decisiones que adopten los decisores. Se hizo una revisión de los estudios más representativos y se estableció orientaciones conceptuales para una mejor comprensión de la diversidad de herramientas matemáticas, estadísticas y aplicativos informáticos disponibles y de mayor uso. El lector podrá acudir a las citas bibliográficas que han sido cuidadosamente seleccionadas para que puedan profundizar en los detalles técnicos. Consideramos que la presente publicación será de interés de los profesionales desarrolladores de proyectos de infraestructuras de disposición final y de los gestores públicos y privados que requieran una orientación preliminar sobre los tipos de modelos de toma de decisiones.

Palabras clave: selección de vertederos, asignación de vertederos, ubicación de vertederos, análisis de decisiones multicriterio, criterios de selección.

\begin{abstract}
The selection of the place where a sanitary landfill will be built is a complex process, which requires the assistance of experts in the final disposal of waste and data analysts, the inclusion of social and political actors as well as the use of support methods to decision making. The objective of this article is to answer the question: What criteria and methods can be used for the selection of a location site in which to build a sanitary landfill? In response, the analysis criteria have been systematized according to socio-economic, environmental, physical and technical factors. A ranking of the most important MCDM methods that can be used by analysts to support decisions made by decision makers has also been made. A review was made of the most representative studies and conceptual guidelines were established for a better understanding of the diversity of mathematical tools, statistics and computer applications available and of greater use. The reader will be able to go to the bibliographic citations that have been carefully selected so that they can delve into the technical details. We believe that this publication will be of interest to professional developers of final disposal infrastructure projects and public and private managers who require preliminary guidance on the types of decision-making models.
\end{abstract}

Keywords: landfill site selection, landfill allocating, landfill siting, multicriteria decision analysis, selection criteria.

\footnotetext{
${ }^{1}$ Universidad Nacional Toribio Rodríguez de Mendoza de Amazonas, Escuela de Pos Grado, Chachapoyas, Perú.

${ }^{2}$ Gerencia Regional de Desarrollo Social, Gobierno Regional Amazonas, Chachapoyas, Perú.

"Autor de correspondencia. E-mail: ccanelounion@hotmail.com
} 


\section{INTRODUCCIÓN}

Las autoridades y directivos en los gobiernos locales cuando asumen funciones se encuentran frecuentemente con graves problemas respecto al manejo y gestión de residuos sólidos urbanos (RSU), no solo porque la disposición final de RSU se realiza inadecuadamente sino porque en la mayoría de los casos se realiza en botaderos no autorizados o ilegales. Dentro de las promesas y compromisos de gestión de muchos de los candidatos está que sin son elegidos establecerán una disposición final adecuada y viable de los RSU de sus comunidades no sólo porque deben cumplir con el imperativo de las normas ambientales sino también porque es un reclamo $\mathrm{y}$ un derecho de su comunidad el vivir en un ambiente saludable y sostenible. Resulta que luego que ya han identificado la tecnología de tratamiento y de disposición final de RSU, deben asumir el gran reto de seleccionar la mejor alternativa de ubicación para la construcción de este tipo de infraestructuras, que cumplan con los requisitos y aborden las expectativas de las partes interesadas y es justamente en esta etapa que existe dificultades para elegir los criterios y las metodologías de análisis espacial y de soporte a la toma de decisiones que los ayuden a escoger la mejor solución.

Para los tomadores de decisión acceder a revisiones pragmáticas y orientativas respecto a que pasos deben cumplir de manera previa a sus decisiones finales resulta una tarea que de por si tiene limitaciones por la diversidad y complejidad de las publicaciones sobre el tema. Por eso, en el presente artículo de revisión el objetivo es presentar de manera práctica los criterios mínimos asociados a la ubicación y las metodologías para la selección de los sitios donde se podrían construir los rellenos sanitarios, teniendo en cuenta eso sí que los modelos matemáticos y estadísticos servirán para sugerir a los decisores cual sería la mejor solución que podrían adoptar dentro de un conjunto de alternativas factibles.

\section{IDENTIFICACIÓN Y PRIORIZACIÓN DE CRITERIOS PARA LA UBICACIÓN DE SITIO DE UN RELLENO SANITARIO}

Una de las preocupaciones debido al efecto NIMBY (Not
In My Back Yard, no en mi patio trasero) es que la puesta en marcha de proyectos de construcción de rellenos sanitarios genere conflictos socio ambientales debido a la percepción contraria de la población a causa de diversas motivaciones y preocupaciones respecto a los posibles impactos en la calidad del aire, disponibilidad de suelo, el valor de la propiedad y los posibles efectos sobre la salud (Gallo, 2019; Brown y Glanz, 2018, Frangopoulos et al., 2018) así como por la rápida difusión de rumores y falsas noticias en las redes sociales (Wang et al., 2021); por eso conviene tomar en cuenta algunas metodologías para el recojo de información de la población que se considera afectada, entre ellas se ha citado al Método de Agrupación de Gray que puede ser complementado con el Método Delphi (Delgado y Romero, 2015) o con la teoría de entropía de Shannon (Delgado y Romero 2016).

Los primeros pasos para la selección del sitio de ubicación de un relleno sanitario son: 1) Identificación preliminar de sitios potenciales luego de la exclusión de áreas inadecuadas, 2) Revisión preliminar de áreas potencialmente adecuadas mediante métodos de selección de sitio, 3) Realización de estudios de selección de sitio detallados de las alternativas y 4) Planeamiento, diseño de ingeniería, costos y la construcción en caso la autoridad competente apruebe el estudio de impacto ambiental (Demesouka et al., 2014).

Para identificar un conjunto mínimo de criterios relevantes (ambientales, sociales y económicos) y evaluar las posibles alternativas de ubicación de un relleno sanitario se requiere el consenso de las opiniones más fiables de los expertos, los actores de la comunidad y las autoridades. Se puede emplear para ello el método Delphi (Romero Collado, 2021), que sirve para extraer y cribar los criterios y subcriterios más importantes luego del consenso de especialistas en lugar de encargar la decisión a un solo experto o autoridad, para posteriormente darles ponderaciones y luego multiplicarles con calificaciones que producen una puntuación numérica cuyo mayor valor es considerado como el más apropiado. El intervalo de 0 a 1 es generalmente adoptado para la normalización, lo que significa que cuanto mayor sea el valor numérico, la idoneidad será mejor. 


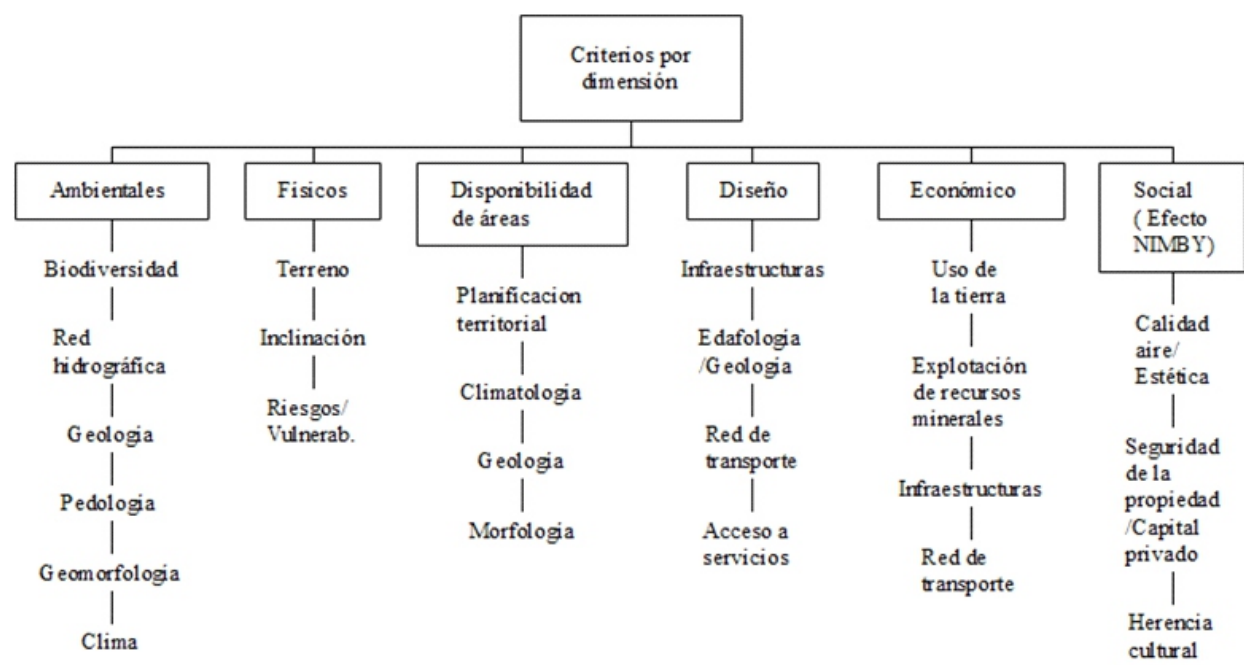

Figura 1. Dimensiones de análisis para la selección del lugar de construcción de un relleno sanitario (modificado de Demesouka et al., 2014).

Escoger los criterios que se deben analizar depende de las regulaciones de cada país, las características de la zona y los diversos factores que componen a la población del ámbito de influencia directo del proyecto. En la Figura 1 se muestra en forma resumida las principales dimensiones que se deben considerar antes de tomar una decisión respecto a la viabilidad técnica y económica de la construcción de un relleno sanitario.

Los criterios más frecuentes que han sido ya estudiados y que bien pueden ser utilizados son: $1=$ Inestabilidad geológica, 2 = Geoformas del área, $4=$ Tipo de suelo, 5 =Vulnerabilidad sísmica, $6=$ Características mecánicas del suelo, 7 = Zona de inundación, $8=$ Uso y ocupación del suelo, $9=$ Pendiente (adecuado de 8 a 12\%, <30\%), $10=$ Permeabilidad del suelo (permeabilidad de $\mathrm{kf}<1 \times$ $10^{-7} \mathrm{~m} / \mathrm{s}$ y espesor $\left.\geq 1 \mathrm{~m}\right), 11=$ Profundidad del agua subterránea $((\geq 10 \mathrm{~m}), 12=$ Distancia de aguas superficiales (lagos, ríos, arroyos, humedales, estanques, embalses), tomas de riego, pozos o suministros de agua ( $\geq 500 \mathrm{~m}$ ), 13= Distancia del relleno a fallas (adecuado $\geq 1000 \mathrm{~m}), 14=$ Distancia a zonas de asentamiento $(\geq 5$ $\mathrm{km}$ de áreas urbanas $\mathrm{y} \geq 1 \mathrm{~km}$ de áreas rurales), $15=$ Densidad poblacional, $16=$ Distancia a zonas de patrimonio cultural $(\geq 1 \mathrm{~km}), 17=$ Sitios turísticos, $18=$ Distancia a sitios ambientales sensibles o protegidos $(\geq$ $500 \mathrm{~m}), 19=$ Servicios básicos, $20=$ Distancia a carreteras o líneas ferroviarias ( $\geq 500 \mathrm{~m}$ ), $21=$ Accesibilidad a la vía, $22=$ Condiciones de la vía de acceso, $23=$ Pendiente de la vía, 24 = Número de vías de acceso, 25 =
Tráfico, $26=$ Costo de transporte desde la zona urbana, $27=$ Distancia a aeropuertos ( $\geq 1.5 \mathrm{~km}), 28=$ Distancia a oleoductos, gasoductos $(>500 \mathrm{~m})$ y tendidos eléctri$\cos (\geq 30 \mathrm{~m}), 29=$ Distancia a instituciones educativas, $30=$ Distancia a instituciones prestadoras de salud, $31=$ Distancia a zonas industriales de alimentos, 32 =Distancia al centro de recolección, 33 = Distancia a granjas, , $34=$ Distancia a materiales de revestimiento o cobertura (sugerido $\leq 5 \mathrm{~km}$ ), $35=$ Calidad del material de revestimiento (índice de plasticidad $\geq 6$, arcillas con un límite líquido del $90 \%$, un índice de plasticidad inferior al $65 \%$ y un contenido de arcilla $>10 \%, \leq 4 \%$ de contracción volumétrica, $\geq 200 \mathrm{kN} / \mathrm{m} 2$ de corte), $36=$ Disponibilidad del material de cobertura ( $\geq 25 \%$ de RSU), 35 $=$ Clima, $36=$ Regulaciones municipales (Rezaeisabzevar et al., 2020; Mohammed et al., 2017; Buenrostro Delgado et al., 2008; Demesouka et al., 2014, Cobos Mora et al., 2021, Fernandez Nascimento et al., 2020). Una vez identificados, definidos y priorizados los criterios y subcriterios de estudio, se debe realizar un análisis multicriterio tanto del problema como de sus alternativas de ubicación, proceso que es sin duda complejo e implica desafíos (Azriati Mat et al., 2017).

\section{LA TOMA DE DECISIONES Y LOS MÉTODOS DE ANÁLISIS MULTICRITERIO}

Tomar una decisión implica escoger la mejor alternativa y asumir sus consecuencias. El enfoque multicriterio sirve para modelar las predilecciones, valores y el juicio 


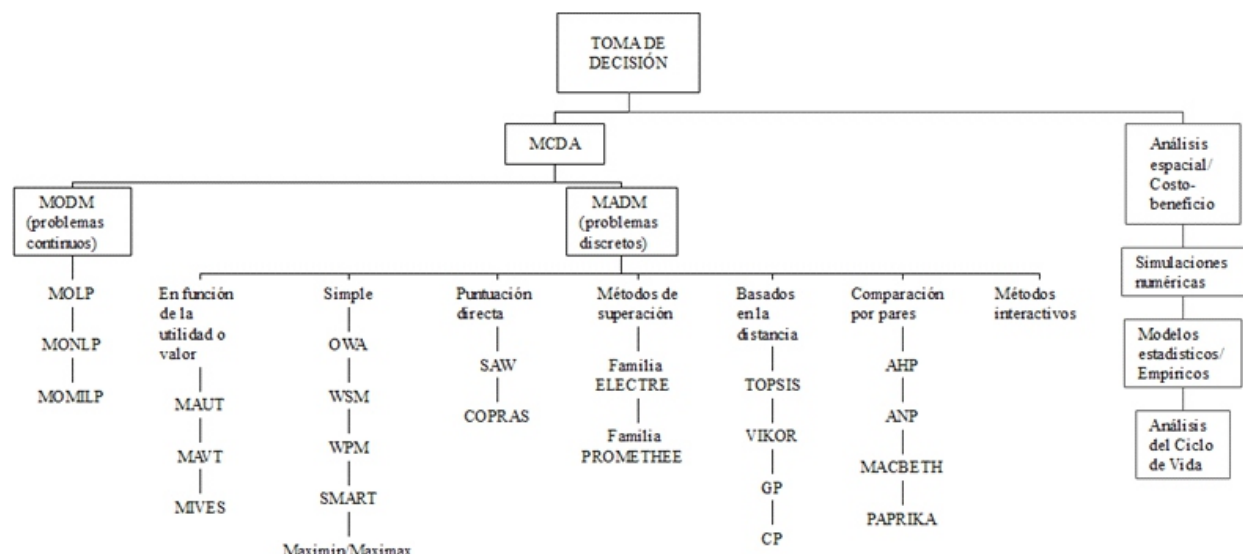

Figura 2. Clasificación de los principales métodos multicriterio (adaptado de Penadés-Plà et al., 2016; Goulart Coelho et al., 2017 y de Hammond et al., 2021).

Nota: AHP = Analytic Hierarchy Process; ANP = Analytic Network Process; $\mathrm{CP}=$ Compromise Programming; GP = Goal Programming; ELECTRE $=$ ELimination and Choice Expressing Reality; $\mathrm{MACBETH}=$ Measuringattractiveness through a Categorical-Based Evaluation Technique; MAUT = Multi-Attribute Utility Theory; MAVT = Multi-Attribute Value Theory; MIVES = Integrated Value Model for Sustainable Evaluations; MOLP = Multi Objective Linear Programming; MONLP = Multi Objective Nonlinear Programming MOMILP = Multi Objective Mixed Integer Linear Programing; OWA = Ordered Weighted Average; PAPRIKA = Potentially All Pairwise Rankings of all Possible Alternatives; PROMETHEE = Preference Ranking Organization Method for Enrichment of Evaluations; SAW = Simple Additive Weighting; SMART $=$ Simple Multi-Attribute Rating Technique; TOPSIS $=$ Technique for Order of Preference by Similarity to Ideal Solution; VIKOR = Multicriterio Optimization and Compromise Solution; WPM = Weighted Product Model; WSM = Weighted Sum Model.

subjetivo de los decisores. El proceso general para tomar una decisión consiste en: 1) Definición del problema, 2) Establecimiento de los requisitos matemáticos o condiciones que deben cumplir las soluciones factibles al problema, 3) Elaboración de los objetivos (intenciones y valores programáticos) que la solución debería alcanzar, 4) Identificación y descripción de las alternativas que cumplan con los requisitos y objetivos. En caso alguna alternativa no cumpla con alguno de los requisitos, se puede optar por descartarla, realizar la modificación o eliminación del requisito o reformular el requisito como objetivo, 5) Definición de los criterios en base a los objetivos. Los criterios deben ser específicos, completos, operativos, no redundantes y en poca cantidad por cada dimensión del problema, 6) Selección del método para la toma de decisiones, 7) Evaluación cuantitativa, cualitativa o combinada de las alternativas en una matriz de decisión multicriterio para la selección de la solución preferida y 8) Validación de la solución escogida de tal manera que cumpla con los requisitos y objetivos (Baker et al., 2001).

Las decisiones que se basan en modelos determinísticos se basan en la certeza de los valores de los factores implicados en consecuencia la prioridad son los resultados. En cambio, en los modelos probabilísticos, los datos se obtienen de manera aleatoria y el que toma las decisiones considera importante tanto a los resultados como el nivel de riesgo que ocasione la decisión que adopte. Los análisis probabilísticos se dividen según las variables aleatorias de estudio en herramientas de tipo discreta o continua, por tanto, los problemas de decisión pueden ser discretos o continuos. En el caso de los discretos comprenden alternativas que son descritas cada una por criterios de evaluación que se miden por valores discretos, numéricos o términos lingüísticos cuya decisión se apoya en el ranking, descripción, clasificación o selección y cuando son de tipo continuo el número de alternativas de solución es infinito pero que en determinadas condiciones pueden fijarse las alternativas aceptables. Más adelante explicaremos sobre los modelos difusos que se emplean en casos cuando el problema está constituido por valores imprecisos que oscilan entre la verdad y la falsedad.

La Toma de Decisiones Multicriterio (Multicriteria Decision Making - MCDM) también conocida como Ayuda a la Decisión Multicriterio (Multiple Criteria Decision Aid - MCDA) sirve para formular modelos explícitos, soluciones adecuadas o viables que generalmente no están completamente normalizados para resolver los problemas y cuyas recomendaciones realizadas por los analistas serán evaluadas por los decisores para que adopten su decisión final sobre la base de la alternativa óptima y más satisfactoria (Rezaeisabzevar et al., 2020). En forma general toda MCDA contempla alternativas realizables, criterios cualitativos o cuanti- 
tativos, la realización de análisis y su principal ventaja en los procedimientos basados en SIG (Sistemas de Información Geográfica) es que los tomadores de decisiones pueden insertar sus preferencias en relación a los criterios de evaluación y alternativas y conocer sus implicancias (Malczewski, 2006). La MCDA se divide en métodos de toma de decisión multi-atributo (Multi Attribute Decision Making - MADM) que sirven para resolver problemas de tipo discreto a través de la elección o clasificación de una cantidad moderada de alternativas predeterminadas y cuando el número de atributos es grande para lo cual los expertos valoran "a priori" cada criterio; en cambio los métodos de toma de decisión multi-objetivo (Multi Objective Decision Making - MODM) se utilizan para dar solución a problemas de tipo continuo referidos a la optimización de diseños (y no de elección) cuando el número de alternativas no predeterminadas es amplio y los expertos participan “a posteriori” (Thies et al., 2019), en la Figura 2 se muestra los principales métodos multicriterio.

\section{PRINCIPALES MCDA PARA LA SELECCIÓN IDÓNEA DEL SITIO DE CONSTRUCCIÓN DE LOS RELLENOS SANITARIOS}

Las técnicas MCDA se pueden emplear para la toma de decisiones individual o grupal, con el empleo de herramientas MODM y MADM y también se pueden clasificar en las que se usan bajo un ambiente de certeza con alternativas y resultados conocidos y constantes cuya alternativa es elegida por su mayor beneficio y las que se realizan bajo incertidumbre con alternativas posibles conocidas y resultados que pueden ser diversos. Bajo un entorno de certidumbre los modelos más frecuentemente empleados son: AHP (Proceso Analítico Jerárquico), ANP (Proceso Analítico de Red), SAW (Ponderación Aditiva Simple), WLC (Combinación Lineal Ponderada), AHP integrada y ANP integrada. En un ambiente de incertidumbre destacan: AHP integrada difusa, ANP integrada difusa, TOPSIS (Técnica de orden de preferencia por similitud a la solución ideal) integrada difusa y VIKOR (Solución de compromiso y optimización de criterios múltiples) integrada difusa (Azriati Mat et al.,
2017). Diversas investigaciones muestran la utilidad, ventajas y desventajas de estas técnicas, que vamos a citar de manera muy general a fin de que los lectores puedan profundizar en los detalles al acudir a las referencias bibliográficas que se ha escogido. La técnica AHP es las más utilizada y conocida para relacionar un problema complejo con su objetivo y para descomponerlo en criterios y subcriterios así como en alternativas con que se cuenta para resolverlo y eso se logra con la construcción de un árbol de jerarquías mediante la elección y colocación de los criterios y subcriterios en una estructura jerárquica descendente mediante la asignación de una ponderación subjetiva según su importancia para luego derivar escalas de razón de comparaciones pareadas tanto discretas como continuas en una matriz cuadrada. Mediante el método convencional de AHP se puede calcular las prioridades para la selección de una o varias alternativas o la ordenación de todas ellas. La escala de intensidad de importancia en el AHP varía de 1 a 9 , donde 1 significa que dos actividades tienen la misma importancia y 9 indica que una actividad respecto a otra es la más importante (R. W. Saaty 1987; Saaty,1994). La técnica AHP con los SIG viene siendo empleada en la ubicación de rellenos sanitarios en muchos lugares , como por ejemplo en Serbia (Zelenovic' Vasiljevi et al., 2012), Makkah (Osra y Kajjumba, 2019), Irak (Alkaradaghi et al., 2019) y tambien con sus versiones combinadas como la AHP-DEMATHEL en Australia (Asefi et al., 2020), la F-AHP en Bangladesh (Islam, Kashem y Morshed 2020), Iran (Chabok et al., 2020; Torabi-Kaveh et al., 2016), Brasil (Faita Pinheiro et al., 2021) y Turquía (Şener y Şener, 2020).

El ANP desarrollado por Saaty (2004), como herramienta de predicción multicriterio proporciona un marco general que ayuda a las decisiones sin que se establezcan suposiciones sobre la independencia de los factores en los distintos niveles, debido a que los elementos se organizan sin un orden particular, es decir sin necesidad de especificar niveles, dominancia o importancia entre los criterios y alternativas (denominados elementos), de tal manera que el ANP está representado por una red de elementos y grupos en lugar de 
una jerarquía lineal y considera a la idea de influencia de unos elementos sobre otros como fundamental, empleando para su cálculo una supermatriz estocástica. ELANP también ha sido de utilidad en los proyectos de construcción de infraestructuras de disposición final, tanto como una metodología unitaria o combinada con WLC en Irán (Karbalaei Saleh et al., 2020) o con DEMATEL en Taiwan (Yu-Ping et al., 2008).

La WLC es uno de los modelos de decisión más utilizados dentro de los SIG por su facilidad de uso para derivar mapas compuestos y por su facil comprensión por parte de los tomadores de decisiones. En este modelo la relación entre objetivos y atributos es de tipo jerárquica (denominada valor estructura), situándose los objetivos generales en el nivel más alto, los cuales se definen por objetivos específicos cuyos indicadores en el nivel más bajo son sus atributos. Esta representación nos proporciona las orientaciones para identificar los mapas de atributos que deben analizarse para posteriormente proceder a su ponderación y seleccionar la alternativa (celda) conveniente (Malczewski, 2000). El mismo autor ha propuesto innovaciones al modelo original al que ha denominado WLC Local que permite generar un mapa de criterios estandarizado (Malczewski, 2011). Su utilidad en la selección de sitio para la ubicación de rellenos sanitarios ha quedado evidenciada en Jordania (Al-Hanbali et al., 2011) y complementado con AHP en Irán (Alizadeh et al., 2013).

Para establecer modelos de idoneidad del sitio los SIG requieren la asignación de un valor de ponderación a sus capas de información geográfica para enfatizar o disminuir su importancia en el cómputo de todo el modelo, una herramienta útil para el analista es la SAW, tambien conocida como el método de combinación lineal ponderada, es la base de la mayoría de técnicas MCDM como la AHP y PROMETHEE, que consiste en realizar a una persona dos pruebas para obtener su opinión sobre la importancia de los criterios. La primera permite la clasificacion de los criterios en orden de importancia y permite al encuestado asignar valores cuantitativos tentativos a los criterios, para ello se apoya en una matriz de comparación por pares y la escala del 1 al 9 de
Saaty. La segunda prueba se apoya en una matriz de decisiones que permite a la persona realizar varias combinaciones de los criterios, logrando de esa manera una asignación más precisa del valor que el individuo otorga a los criterios. El procedimiento se puede realizar con varias personas y los resultados se promedian y normalizan para lograr a un consenso (Churchman y Ackoff 1954, Afshari et al., 2010, Memariami et al., 2009). La SAW ha sido de utilidad en la toma de decisiones de ubicación de rellenos sanitarios en Irán (Mokhtari et al., 2016) y Turquía (İzzeddin Karabulut et al., 2021), combinado con AHP en Irak (Jalil Chabuk et al., 2017) y Colombia (Mejía et al., 2012).

El modelo TOPSIS ha sido diseñado para la selección de la mejor alternativa frente a un número finito de criterios, con la condición de tener la menor distancia ante la solución ideal positiva y la distancia más lejana respecto a la solución ideal negativa. La condición es que los valores de los atributos sean numéricos lo que permite que las alternativas viables se puedan ordenar de manera descendente según su coeficiente de proximidad (Behzadian et al., 2012).

La técnica VIKOR (VlseKriterijumska Optimizacija I Kompromisno Resenje) ha sido desarrollada para resolver problemas discretos de criterios múltiples que tienen distintas unidades o por ser de tipo cualitativo y cuantitativo. Las alternativas al problema se clasifican y seleccionan de un conjunto de alternativas (clasificación de compromiso) de acuerdo a la distancia más cercana a la alternativa de solución ideal (solución de compromiso) la cual debe ser la de mayor ventaja y estabilidad y que sería la aceptada por los tomadores de decisión (Opricovic y Tzeng, 2004). VIKOR tiene cada vez más áreas de aplicación que incluye la gestión de la construcción, toma de decisiones, sostenibilidad y energías renovables (Mardani et al., 2016). El VIKOR basado en actitudes (ITL-VIKOR) ha sido empleado teniendo en cuenta la actitud pesimista u optimista de los decisores en la selección de sitio de una planta de combustión en Estambul (Hu-Chen et al., 2014).

La toma de decisiones en situaciones de subjetividad, vaguedad, incertidumbre, ambigüedad, imprecisión o 
razonamiento incompleto se puede abordar desde la lógica difusa, borrosa o la teoría de los conjuntos borrosos, cuyo símbolo F (del inglés fuzzy) será empleado para su identificación. La teoría difusa va más allá de la lógica clásica aristotélica bivalente o las probabilidades para dar paso a las posibilidades y aproximarse al entendimiento del mundo real como lo hacen los humanos en el cual los fenómenos son casi todos una cuestión de grado, es decir de generalizaciones de conceptos, teorías, algoritmos y formalismos así como de una tolerancia a la imprecisión que incluye también el uso de escalas lingüisticas lo que ayuda a la simplificación del modelo de análisis y por ende se reducen sus costos (Zadeh, 1996 y 2015; Castiblanco Ruiz, 2013). La teoría difusa es ahora empleada con las tecnologías SIG para la generación de escenarios de decisión (Jiang y Eastman, 2000), El F-AHP se emplea ampliamente en la administración, industria y en lo ambiental, en la generación de mapas de idoneidad para la ubicación de rellenos (Gemitzi et al., 2007), dentro del entorno de la herramienta MATLAB (Nazari et al., 2012), con modificaciones como la F-AHP pitagórica (Karasan, Ilbahar y Kahraman 2019) o en combinaciones tales como la FAHP-TOPSIS en la India (Govind Kharat et al., 2016) y Turquía (Beskese et al., 2015). Un análisis comparativo de los métodos F-TODIM y F- AHP difuso ha sido realizado por Hanine et al. (2016). La F-ANP tambien es de utilidad en la ubicación de instalaciones de disposición final (Isalou et al., 2012). La F-TOPSIS tiene un gran potencial en los problemas de localización de inversiones (Nadaban et al., 2016), soluciones respetuosas con el medio ambiente (Palczewski y Sałabun, 2019) y la TOPSIS hibrida en la disposición de de residuos (Roy et al., 2016). La metodología IVF-VIKOR que se basa en la incertidumbre y el riesgo ha sido empleada para la selección de una ubicación adecuada para residuos húmedos (Mokhtarian et al., 2014).

En diversos estudios ha quedado demostrado que los métodos MADM son los más utilizados, teniendo potencial también los modelos MODM. Los más frecuentemente reportados para la ubicación de rellenos sanitarios son el AHP, ANP, MAVT / MAUT y TOPSIS y para la gestión de residuos PROMETHEE y ELECTRO (Goulart Coelho, Lange y Coelho 2017), en combinación frecuente con los SIG (Donevska et al., 2021). La gran variedad de campos de aplicación del AHP por las organizaciones públicas y privadas contribuye a su mayor empleo, incluso en combinación con otras técnicas como la teoría difusa, TOPSIS, MIVES y TRIZ (Dos Santos et al., 2019). El Método Delphi es ampliamente usado para analizar las opiniones de los actores sociales aún cuando puede también tener ciertas limitaciones (Sierra et al., 2018). Contar con información adecuada y suficiente permitirá a las autoridades diseñar las mejores estrategias para forjar acuerdos con la población lo que a su vez disminuirá los costos sociales y económicos. Los enfoques antes mencionados podrían mejorar su aplicabilidad si incluyeran en su desarrollo aspectos relacionados a la viabilidad económica y vida útil del proyecto (Sadek et al., 2006). Finalmente se prevee que la selección de sitio tambien incluirá la evaluación de riesgos mediante la combinación de SIG, nuevas herramientas híbridas de MCDA e inteligencia artificial (Kuba Nuhu et al., 2021).

\section{CONCLUSIONES}

Para la identificación y selección de criterios de análisis de ubicación de un relleno sanitario se debe considerar como mínimo las dimensiones: social, económica, física y ambiental, para lograrlo se pude emplear el método Delphi y la revisión de la información técnicocientífica y normativa. El efecto NIMBY y la representación social ante ante los proyectos de construcción puede estudiarse con la ayuda del Método de Agrupación de Gray o con la teoría de entropía de Shannon. La elección de la alternativa más adecuada para la ubicación de la infraestructura de disposición final es un proceso complejo que requiere el apoyo con métodos MCDA que pueden emplearse de manera sola o en forma híbrida entre ellos, con las herramientas SIG, el análisis de ciclo de vida o con la Teoría Difusa. En todo el proceso de análisis es necesario que los procedimientos de normalización de datos y evaluación de sensibilidad sean explicados para conocer su fiabilidad. 


\section{CONTRIBUCIÓN DE LOS AUTORES}

El autor participó en la redacción del manuscrito inicial, revisión bibliográfica, y en la revisión y aprobación del manuscrito final.

\section{CONFLICTO DE INTERESES}

El autor declara no tener conflicto de intereses.

\section{REFERENCIAS BIBLIOGRÁFICAS}

Afshari, A., M. Mojahed, y R. Mohd Yusuff . 2010. “Simple Additive Weighting approach to Personnel Selection problem". International Journal of Innovation, Management and Technology 1 (5): 511-515.

Al-Hanbali, A., B. Alsaaideh, y A. Kondoh. 2011. "Using GIS-Based Weighted Linear Combination Analysis and Remote Sensing Techniques to Select Optimum Solid Waste Disposal Sites within Mafraq City, Jordan". Journal of Geographic Information System 3: 267-278.

Alizadeh, M., I. Ngah, H. Shahabi, y E. Ali zadeh. 2013. "Evaluating AHP and WLC Methods in Site Selection of Waste Landfill (Case Study: Amol, North of Iran)". J. Basic. Appl. Sci. Res. 3 (5): 83-88.

Alkaradaghi, Karwan, Salahalddin S Ali, Nadhir AlAnsari, Jan Laue, y Ali Chabuk. 2019. "Landfill Site Selection Using MCDM Methods and GIS in the Sulaimaniyah Governorate, Iraq". Sustainability 11 (17): 1-22.

Asefi, H., Y. Zhang, S. Lim, M. Maghrebi, y S. Shahparvari. 2020. "A multi-criteria decision support framework for municipal solid waste landfill siting: a case tudy of New South Wales (Australia)". Environmental Monitoring and Assessment 192: 682.

Azriati M., A. M. Benjamin, y S. Abdul-Rahman. 2017

"A review on criteria and decision-making techniques in solving landfill site selection problems". Journal of Advanced Review on Scientific Research 37 (1):14-32.

Babalola, A. y I. Busu. 2011. "Selection of Landfill Sites for Solid Waste Treatment in Damaturu TownUsing GIS Techniques". Journal of Environmental Protection 2: 1-10.
Baker, D., D. Bridges, R. Hunter, G. Johnson, J. Krupa, J. Murphy, y K. Sorenson. 2001. Guidebook to decision-makig methods. Washington D.C. (EEUU): Department of Energy.

Behzadian, M., S. K. Otaghsara, M. Yazdani, y J. Ignatius. 2012. "A state-of the-art survey of TOPSIS applications". Expert Systems with Applications 39: 13051-13069.

Beskese, A., H. Handan Demir, H. Kurtulus Ozcan, y H. Eser Okten. 2015. "Landfill site selection using fuzzy AHP and fuzzy TOPSIS: a case study for Istanbul". Environ Earth Sci 73: 3513-3521.

Brown, G. y H. Glanz. 2018. "Identifying potential NIMBY and YIMBY effects in general land use planning and zoning". Applied Geography 99: 1-11.

Buenrostro Delgado, O., M. Mendoza, E. López Granados, y D. Geneletti. 2008. “Analysis of land suitability for the siting of inter-municipal landfills in the Cuitzeo Lake Basin, Mexico". Waste Management 28: 1137-1146.

Castiblanco Ruiz, F. A. 2013. "La incertidumbre y la subjetividad en la toma de decisiones: una revisión desde la lógica difusa". Lúmina 14: 116-140.

Chabok , M., A. Asakereh, H. Bahrami y N. Ollah Jaafarzadeh. 2020. "Selection of MSW landfill site by fuzzy-AHP approach combined with GIS: case study in Ahvaz, Iran”. Environ Monit Assess 192 (433): 1-15.

Cheng, E. W. y H. Li. 2005. “Analytic Network Process Applied to Project Selection". Journal of Construction Engineering and Management 131 (4): 459-466.

Churchman, C. W. y R. Ackoff. 1954. “An Approximate Measure of Value". Journal of the Operations Research Society of America 2 (2): 172-187.

Cobos M., S. Lucía, J. L. Solano Peláez, y P. C. Gárate Rodríguez. 2021 "Criterios de selección para un sitio de disposición final de residuos". Rev. Int. Contam. Ambie. 37: 39-53.

Delgado, A. y I. Romero. 2016. "Environmental conflict analysis using an integrated grey clustering and entropy-weight method: A case study 
of a mining project in Peru". Environmental Modelling \& Software 77: 108-121.

Delgado, A. y I. Romero. 2015. "Selección de un método para la evaluación del impacto social usando AHP”. Revista ECIPerú 12 (1): 84-91.

Demesouka, O. E., A. P. Vavatsikos, y K. P. Anagnostopoulos. 2014. "GIS-based multicriteria municipal solid waste landfill suitability analysis: A review of the methodologies performed and criteria implemented". Waste Management \& Research 32 (4): 270-296.

Donevska, K., J. Jovanovski, y L. Gligorova. 2021. "Comprehensive Review of the Landfill Site Selection Methodologies and Criteria”. J. Indian Inst. Sci. 101: 509-521.

Dos Santos, P. H., S. Miranda Neves, D. Ornaghi Sant'Anna, C. Henrique de Oliveira, y H. Duarte Carvalho. 2019. "The analytic hierarchy process supporting decision making for sustainable development: An overview of applications". Journal of Cleaner Production 212 (2019): 119-138.

Faita Pinheiro, M. M. , L. Prado Osco, T. Sussel Gonçalves Mendes, R. Ennes Cicerelli, y A. Paula Marques Ramos. 2021. "Uma nova estratégia para definir áreas para aterros sanitários consorciados baseados em análise multicritério". Revista Brasileira de Geografia Física 14 (1): 420-430.

Fernandez Nascimento, V., A. Isabel Silva Loureiro, P. R. Andrade, L. Antonio Guasselli, y J. Pierre Balbaud Ometto. 2020. "A worldwide metaanalysis review of restriction criteria for landfill siting using geographic information systems". Waste Management \& Research 39 (3): 1-18.

Frangopoulos, Y., E. Kolovou, y D. Kourkouridis. 2018. "N.I.M.B.Y. Syndrome and Landfills: The case of the landfills in the Region of Pella and the emergence of a NIMBY syndrome". Modern Management Forum 3 (2): 1-12.

Gallo, M. 2019. “An Optimisation Model to Consider the NIMBY Syndrome within the Landfill Siting Problem”. Sustainability 11: 3904.

Gemitzi, A., V. A. Tsihrintzis, E. Voudrias, C. Petalas, y G. Stravodimos. 2007. “Combining geograp- hic information system, multicriteria evaluation techniques and fuzzy logic in siting MSW landfills". Environ Geol 51: 797-811.

Goulart C., M. Lineker, L. C. Lange, y H. M. G. Coelho. 2017. "Multi-criteria decision making to support waste management: A critical review of current practices and methods". Waste Management \& Research 35 (1): 3-28.

Govind Kharat, M., S. Jaisingh Kamble, R. D. Raut, S. S. Kamble, y S. M. Dhume. 2016. "Modeling landfill site selection using an integrated fuzzy MCDM approach”. Model. Earth Syst. Environ 2 (53): 1-16.

Habiba, I. M., M. Zulkepli, B. Yuso Norhakim, y B. Yamusa Yamusa . 2018. "Analysis of MultiCriteria Evaluation Method of Landfill Site Selection for Municipal Solid Waste Management". E3S Web of Conferences 34: 02010.

Hammond, E. B., F. Coulon, S. H. Hallett, R. Thomas, D. Hardy, y A. Kingdon. 2021. "A critical review of decision support systems for brownfield redevelopment'. Science of the Total Environment 785: 147132.

Hanine, M., O. Boutkhoum, A. Tikniouine, y T. Agouti. 2016. "Comparison of fuzzy AHP and fuzzy TODIM methods for landfill location selection”. SpringerPlus 5: 501.

Hu-Chen, L., Y. Jian-Xin , F. Xiao-Jun, y C. Yi-Zeng. 2014. "Site selection in waste management by the VIKOR method using linguistic assessment". Applied Soft Computing 21: 453-461.

Ibrahim Mohammed, H., Z. Majid, N. Bin Yusof, y Y. Bello Yamusa. 2019. "A Review of GIS-Based and Multi-criteria Evaluation Method for Sustainable Landfill Site Selection Model". En Global Civil Engineering Conference. Pradhan, B. (ed). Singapur (Singapur): Springer Nature.

Isalou, A. A., V. Zamani, B. Shahmoradi, y H. Alizadeh. 2012. "Landfill site selection using integrated fuzzy logic and analytic network process (FANP)". Environ Earth Sci 68 (6): 1745-1755.

Islam, M., S. Kashem, y S. Morshed. 2020. "Integrating spatial information technologies and fuzzy analytic hierarchy process (F-AHP) approach for landfill 
siting". City and Environment Interactions 7: 1-14.

İ. K., Abdullah , B. Yazici-Karabulut, P. Derin, M. Irfan Yesilnacar, y M. Ali Cullu. 2021. 'Landfill siting for municipal solid waste using remote sensing and geographic information system integrated analytic hierarchy process and simple additive weighting methods from the point of view of a fast-growing metropolitan area in GAP area of Turkey". Environmental Science and Pollution Research.

Jalil Chabuk, A., N. Al-Ansari, H. Musa Hussain, S. Knutsson, y R. Pusch. 2017. “GIS-based assessment of combined AHP and SAW methods for selecting suitable sites for landfill in Al-Musayiab Qadhaa, Babylon, Iraq". Environ Earth Sci 76: 209.

Jiang, H. y J. Ronald Eastman. 2000. “Application of fuzzy measures in multi-criteria evaluation in GIS". International Journal of Geographical Information 14(2): 173-184.

Karasan, A., E. Ilbahar, y C. Kahraman. 2019. “A novel pythagorean fuzzy AHP and its application to landfill site selection problem". Soft Computing 23: 10953-10968.

Karbalaei Saleh, S., H. Aliani, y S. Amoushahi. 2020. "Application of modeling based on fuzzy logic with multi-criteria method in determining appropriate municipal landfill sites (case study: Kerman City)". Arabian Journal of Geosciences 13: 1219.

Kuba Nuhu, S., Z. Abdul Manan, S. Rafidah Wan Alwi, y M. Nadzri Md Reba. 2021. "Roles of geospatial technology in eco-industrial park site selection: State of the art review". Journal of Cleaner Production 309: 127361.

Liu, J., Y. Li, B. Xiao, y J. Jiao. 2021. “Coupling Fuzzy Multi-Criteria Decision-Making and Clustering Algorithm for MSW Landfill Site Selection (Case Study: Lanzhou, China)". Int. J. Geo-Inf. 10: 403.

Malczewski, J. 2006. “GIS-based multicriteria decision analysis: a survey of the literature". International Journal of Geographical Information Science 20 (7): 703-726.

Malczewski, J. 2011. "Local Weighted Linear Combination". Transactions in GIS 15 (4): 439-455.

Malczewski, J. 2000. "On the Use of Weighted Linear Com- bination Method in GIS: Common and Best Practice Approaches". Transactions in GIS 4 (1): 5-22.

Mardani, A., E. Kazimieras Zavadskas, K. Govindan, A. Amat Senin, y A. Jusoh. 2016. "VIKOR Technique: A Systematic Review of the State of the Art Literature on Methodologies and Applications". Sustainability 8(37): 1-38.

Memariami, A., A. Amini, y A. Alinezhad. 2009. "Sensitivity Analysis of Simple Additive Weighting Method (SAW): he Results of Change in the Weight of One Attribute on the Final Ranking of Alternatives". Journal of Industrial Engineering 4: 13-18.

Mohammed, H. I., Z. Majid, N. B. Yusof, y Y. B. Yamusa. 2017. "GIS-based an multi-criteria evaluation method for optimised landfill site". Proceeding of the 1st global civil engineering conference.

Mokhtari, E., M. Khamehchian, G. Montazer, y M. Nikudel. 2016. "Landfill site selection using Simple Additive Weighting (SAW)". International Journal of Geography and Geology 3 (10): 209-223.

Mokhtarian, M. N., S. Sadi-nezhad, y A. Makui. 2014. “A new flexible and reliable interval valued fuzzy VIKOR method based on uncertainty risk reduction in decision making process: An application for determining a suitable location for digging some pits for municipal wet waste landfill'. Computers \& Industrial Engineering 78: 213-233.

Nadaban, S., S. Dzitac, y I. Dzitac. 2016. "Fuzzy TOPSIS: A General View". Procedia Computer Science 91: 823-831.

Nazari, A., M. Mehdi Salarirad, y A. Aghajani Bazzazi. 2012. "Landfill site selection by decision-making tools based on fuzzy multi-attribute decision-making method". Environ Earth Sci 65: 1631-1642.

Opricovic, S. y G. H. Tzeng. 2004. "Compromise solution by MCDM methods: A comparative analysis of VIKOR and TOPSIS". European Journal of Operational Research 156: 445-455.

Osra, F. A. y G. W. Kajjumba. 2019. “Landfill site selection in Makkah using geographic information system and analytical hierarchy process". Waste Management \& Research 38 (3): 245-253. 
Palczewski, K., y W. Sałabun. 2019. "The fuzzy TOPSIS applications in the last decade". Procedia Computer Science 159: 2294-2303.

Penadés-Plà, V., T. García Segura, J. V. Martí, y V. Yepes. 2016. "A review multicriteria decision making methods applied to the sustanaible bridge design”. Sustainabilily 8 (12): 1295.

Rezaeisabzevar, Y., A. Bazargan, y B. Zohourian. 2020. "Landfill site selection using multicriteria decision making: Influential factors for comparing locations". Journal of Environmental Sciences 93: 170-184.

Rezaeisabzevar, Y., A. Bazargan, y B. Zohourian. 2020. "Landfill site selection using multi criteria decision making: Influential factors for comparing locations". Journal of Environmental Science 93: 170-184.\}

Romero Collado, A. 2021. "Essential elements to elaborate a study with the (e)Delphi method". Enfermería intensiva 32: 100-104.

Roy, J., K. Adhikary, y S. Kar. 2016. Artificial intelligence. https://arxiv.org/abs/1606.08965 (Consultada el 5 de junio de 2021).

Saaty, R W. 1987. “The Analytic Hierarchy Process What it is an how it is used". Mathl Modelling 9(3-5): 161-176.

Saaty, T. L. 2004. "Fundamentals of the Analytic Network Process dependence and feedback in decisionmaking with a single network". Journal of Systems Science and Systems Engineering 13 (2): 129-157.

Saaty, T. L. 1994. "How to Make a Decision: The Analytic Hierarchy Process". Interfaces 24 (6): 19-43.

Sadek, S., M. El-Fadel, y F. Freiha. 2006. "Compliance factors within a GIS-based framework". International Journal of Environmental Studies 63 (1): 71-86.

Şener, E., y Ş. Şener. 2020. "Landfill site selection using integrated fuzzy logic and analytic hierarchy process (AHP) in lake basins". Arabian Journal of Geosciences 13: 1130.

Sierra, L. A., V. Yepes, y E. Pellicer. 2018. “A review of multi-criteria assessment of the social sustainability of infrastructures". Journal of
Cleaner Production 187: 496-513.

Thies, C., K. Kieckhäfer, T. S. Spengler, y M. S. Sodhi. 2019. "Operations research for sustainability assessment of products: A review". European Journal of Operational Research 274: 1-21.

Torabi-Kaveh, M., R. Babazadeh, S. D. Mohammadi, y M. Zaresefat. 2016. "Landfill site selection using combination of GIS and fuzzy AHP, a case study: Iranshahr, Iran". Waste Management \& Research 34 (5): 438-448.

Tuzkaya, G., S. Önüt, U. R. Tuzkaya, y B. Gülsün. 2008. "An analytic network process approach for locating undesirable facilities: An example from Istanbul, Turkey". Journal of Environmental Management 88: 970-983.

Wang, Y., L. Zheng, y J. Zuo. 2021. “Online rumor propagation of social media on NIMBY conflict: Temporal patterns, frameworks and rumor-mongers". Environmental Impact Assessment Review 91: 106647.

Yu-Ping, O. Y., S. How-Ming, L. Jun-Der, y T. GwoHshiung. 2008. “A Novel Hybrid MCDM Model Combined with DEMATEL and ANP with Applications". International Journal of Operations Research 5(3): 160-168.

Zadeh, L. A. 2015. "Fuzzy logic - a personal perspective". Fuzzy Sets and Systems 281: 4-20.

Zadeh, L. A. 1996. "Nacimiento y evolución de la lógica borrosa, el soft computing y la computación con palabras: un punto de vista personal". Psicothema 8 (2): 421-429.

Zafra Mejía, C. A., F. Andrés Mendoza Castañeda, y P. Alejandra Montoya Varela. 2012. “A methodology for landfill location using geographic information systems: a Colombian regional case". Ingeniería e Investigación 32 (1): 64-70.

Zelenovic' Vasiljevic, T., Z. Srdjevic, R. Bajc`etic', y M. Vojinovic' Miloradov. 2012. "GIS and the Analytic Hierarchy Process for Regional Landfill Site Selection in Transitional Countries: A Case Study From Serbia". Environmental Management 49: 445-458. 